Revta brasil. Bot., São Paulo, V.24, n.3, p.235-248, set. 2001

\title{
Similarity among periphyton algal communities in a lentic-lotic gradient of the upper Paraná river floodplain, Brazil
}

\author{
LILIANA RODRIGUES ${ }^{1,3}$ and DENISE DE C. BICUDO ${ }^{2}$
}

(received: September 13, 2000; accepted: April 4, 2001)

\begin{abstract}
Similarity among periphyton algal communities in a lentic-lotic gradient of the upper Paraná river floodplain, Brazil). Floristic comparison of periphyton communities from three systems with different hydrodynamic regimes (lentic, semilotic, and lotic) was carried out during high and low water periods on the Upper Paraná River floodplain. For each period and system, glass slides were sampled every two days during 18-day periods, and Eichhornia azurea Kunth petioles were sampled three times. A total of 228 species was collected, representing 12 classes, mainly diatoms and desmids. The highest species-richness was found in communities from lentic system and during high water. Species richness in the lotic system was more stable over succession and hydrological periods. Algal taxonomic structure in river community was clearly separated from the other two systems, with $43 \%$ of similarity level. The hydrological period was next in importance, followed last by the substratum type, with communities associated at $65-78 \%$ similarity levels, depending on system and hydrological period. The type of system, but not the water levels,was the main factor that influenced community richness, followed by disturbances caused by flood pulses and the operation of reservoirs upstream. The periphyton on artificial and natural substrata presented high degree of similarity.
\end{abstract}

RESUMO - (Similaridade entre comunidades de algas perifíticas em um gradiente lêntico-lótico da planície de inundação do alto rio Paraná, Brasil). Comparação florística entre as comunidades perifíticas de três sistemas com distintos regimes hidrodinâmicos (lêntico, semilótico e lótico), abrangendo períodos de águas altas e baixas, foi realizada a partir de substratos artificial e natural. Lâminas de vidro foram coletadas a cada dois dias durante 18 dias e pecíolos de Eichhornia azurea Kunth três vezes em cada ocasião. O perifíton apresentou, como um todo, 228 espécies de algas distribuídas em 12 classes, com a maior representatividade de diatomáceas e desmídias. Maior riqueza foi, invariavelmente, encontrada no sistema lêntico e no período de águas altas. A ficoflórula perifítica foi caracterizada, principalmente, pelo regime hidrodinâmico e pela morfometria do ambiente, havendo nítida separação da comunidade do sistema lótico em relação à dos outros dois sistemas, agrupados a $43 \%$ de similaridade. Em seguida, o período hidrológico foi mais importante e, por último, o tipo de substrato, cujas comunidades se associaram a $65-78 \%$ de similaridade, dependendo do sistema e período hidrológico. Conclui-se que o tipo de ambiente e não o regime hidrológico exerceu a maior influência sobre a riqueza específica da comunidade; e que as perturbações causadas pelos pulsos de inundação e pela operação dos reservatórios influenciaram, marcadamente, as flutuações da riqueza específica das comunidades perifíticas. Finalmente, as comunidades epifíticas foram floristicamente bem representadas pelos substratos artificiais.

Key words - Periphyton, benthic algae, species composition, similarity, tropical floodplain

\section{Introduction}

The floodplains are among the most productive ecosystems of the globe and are also important centers of biological diversification (Junk 1996). Large undammed tropical rivers typically support floodplains leading to high habitat heterogeneity. Such systems are characterized by the presence of different types of aquatic and transitional land-water habitats such as oxbow lakes, temporary lakes, semilotic environments, streams, and rivers. These

1. Universidade Estadual de Maringá (UEM) - DBI/NUPELIA/ PEA, Av. Colombo, 5790, 87020-900 Maringá, PR, Brasil.

2. Instituto de Botânica, Seção de Ecologia, Caixa Postal 4005, 01061-970, São Paulo, SP, Brasil.

3. Corresponding author: 1rodrigues@wnet.com.br mostly shallow systems usually support extensive banks of aquatic macrophytes, which provide conditions for a profuse development of periphytic and metaphytic algae. In flooded areas in general, these communities interact closely with phytoplankton, mainly through export of matter from the substratum to open water (Goldsborough \& Robinson 1996).

Despite the abundance of wetland habitat globally, information on the algal assemblages of wetlands remains fragmentary, particularly in subarctic and tropical areas (Goldsborough \& Robinson 1996). Although the extensive area of floodplains occupied in Brazil (Junk \& Da Silva 1995) and the importance of attached microbiota in shallow systems, very little work has been done on periphyton in such systems in Brazil (Bicudo et al. 1995). 
The floodplain of the Upper Paraná River, the object of this study, is located in the last undammed section of this river in Brazil (Agostinho \& Zalewski 1996). In spite of the incontestable importance of algae, knowledge of such communities in the Upper Paraná River floodplain has lagged behind that of physical and chemical limnology and studies of other communities, as clearly documented by the review of Vazzoler et al. (1997). Taxonomic and ecological information have been published for phytoplankton in this system (Jati \& Train 1993, Train \& Rodrigues 1997) but information on periphyton is lacking.

Considering the extremely heterogeneous distribution of the periphyton and its interaction with natural substrata, allied to the necessity to standardize the community development time and the nature of the substratum, there has been an incentive for the use of artificial substrata in spatial and temporal comparisons, mainly in biomonitoring studies (Cattaneo \& Amireault 1992).

This investigation is part of a broader project on successional development of periphyton communities on artificial substrata. The objectives of the present study were: a) to compare the flora of the periphyton community of three systems with distinct hydrodynamic regimes (lentic, semilotic, and lotic) and in two hydrological periods (high and low water); b) to evaluate the efficiency of the use of artificial substrata (glass slides) in representing the epiphytic algal flora in the Upper Paraná River floodplain; and c) to provide taxonomic support for parallel ecological studies. This work is a pioneering contribution to the study of the periphyton flora of this segment of the plain. It is also the first to provide floristic comparisons of the periphyton algal communities of different systems and substrata (natural/artificial) in a Brazilian floodplain.

\section{Material and methods}

Study Area - The upper Paraná River flows generally from North to South/Southwest through a region with tropical to subtropical climate, with mean monthly temperatures above $15{ }^{\circ} \mathrm{C}$ and precipitation over 1,500 mm.year ${ }^{-1}$ (Agostinho \& Zalewski 1996). The work was carried out near the municipality of Porto Rico, state of Paraná, at the boundary with the state of Mato Grosso do Sul, between coordinates $22^{\circ} 40^{\prime}-22^{\circ} 45^{\prime} \mathrm{S}$ and $53^{\circ} 15^{\prime}-53^{\circ} 25^{\prime} \mathrm{W}$ (figure 1 ).

Three aquatic systems with distinct hydrodynamic regimes were chosen. Garças Lake is a lentic environment, which communicates permanently to the river through a narrow channel and is about $2 \mathrm{~km}$ long and $150 \mathrm{~m}$ wide. Its littoral zone harbors a species-rich community of aquatic macrophytes, with Eichhornia azurea Kunth prominent, and arboreal riparian vegetation extends along nearly its entire shoreline. Pau Véio Backwater, located on Mutum Island, state of Paraná, is a semilotic environment about $1.2 \mathrm{~km}$ long and $50 \mathrm{~m}$ wide. Its left bank is formed by a natural levee, which separates it from the Paraná River. The vegetation grades from aquatic to terrestrial systems and is composed of aquatic macrophytes, mainly E. azurea, and herbaceous and shrubby riparian vegetation; the right bank is mainly in pasture. Cortado Channel, a lotic environment located on the left bank of the Paraná River, is about $2 \mathrm{~km}$ long, $80 \mathrm{~m}$ wide and mean water current of 50 and $30 \mathrm{~cm} . \mathrm{s}^{-1}$ in high and low water, respectively. It has arboreal riparian vegetation along both banks, and there are multispecies stands of aquatic macrophytes, mainly E. azurea, in the littoral zone (figure 1).

Characterization of the abiotic limnological parameters of these systems was carried out simultaneously with the present study. Data were thoroughly discussed by Rodrigues \& Bicudo (2001) and some basic information are provided in table 1 and figure 2 .

Sampling was carried out during periods of high water (March 16, through April 1, 1994) and low water (July 20, through August 5, 1994) in the three study systems. For purposes of comparison, the type of substratum and the stage of development of the periphyton community were standardized. Samples for taxonomic characterization of periphyton communities were taken from glass slides as an artificial substratum, and petioles of Eichhornia azurea Kunth in the mature stage, as this macrophyte was best represented in all three environments. Wooden supports containing vertically positioned glass slides were placed in the littoral zones of the three systems, next to stands of E. azurea.

Collections of periphyton from glass slides were made every two days during 18-day periods in high and low water phases (figure 2), with two replicates and with random sampling of the substrata. Natural substrata were sampled three times, corresponding to the $2^{\text {nd }}, 10^{\text {th }}$, and $18^{\text {th }}$ day of successional development on the artificial substrata. Natural substrata were sampled to study the community under natural conditions and in a more stationary mature developmental stage.

The periphyton removed from a substratum was fixed and preserved in $0.5 \%$ acetic lugol, except for samples for determination of diatoms, which were preserved in $4 \%$ formalin. Techniques of oxidization and preparation of permanent slides of diatoms followed Simonsen (1974), using HYRAX as the inclusion medium. Samples were archived in the Herbarium of the State University of Maringá, Paraná (HUM).

After taxonomic analysis, organisms were quantified using an inverted microscope at $400 \mathrm{X}$, according to methods of Utermöhl (1958). Additional counts were made on permanent slides (100 individuals) to differentiate very similar diatom species as recommended by Biggs (1996). Evaluation of floristic similarities was based on the quantification procedure for purposes of standardizing the sampling and the analyses efforts among periods and systems.

The classification system used was that of Round (1968), complemented by Round (1971). The systematic arrangement and generic diagnoses followed Bourrelly $(1975,1981)$, except for the classes Cyanophyceae and Bacillariophyceae, which 


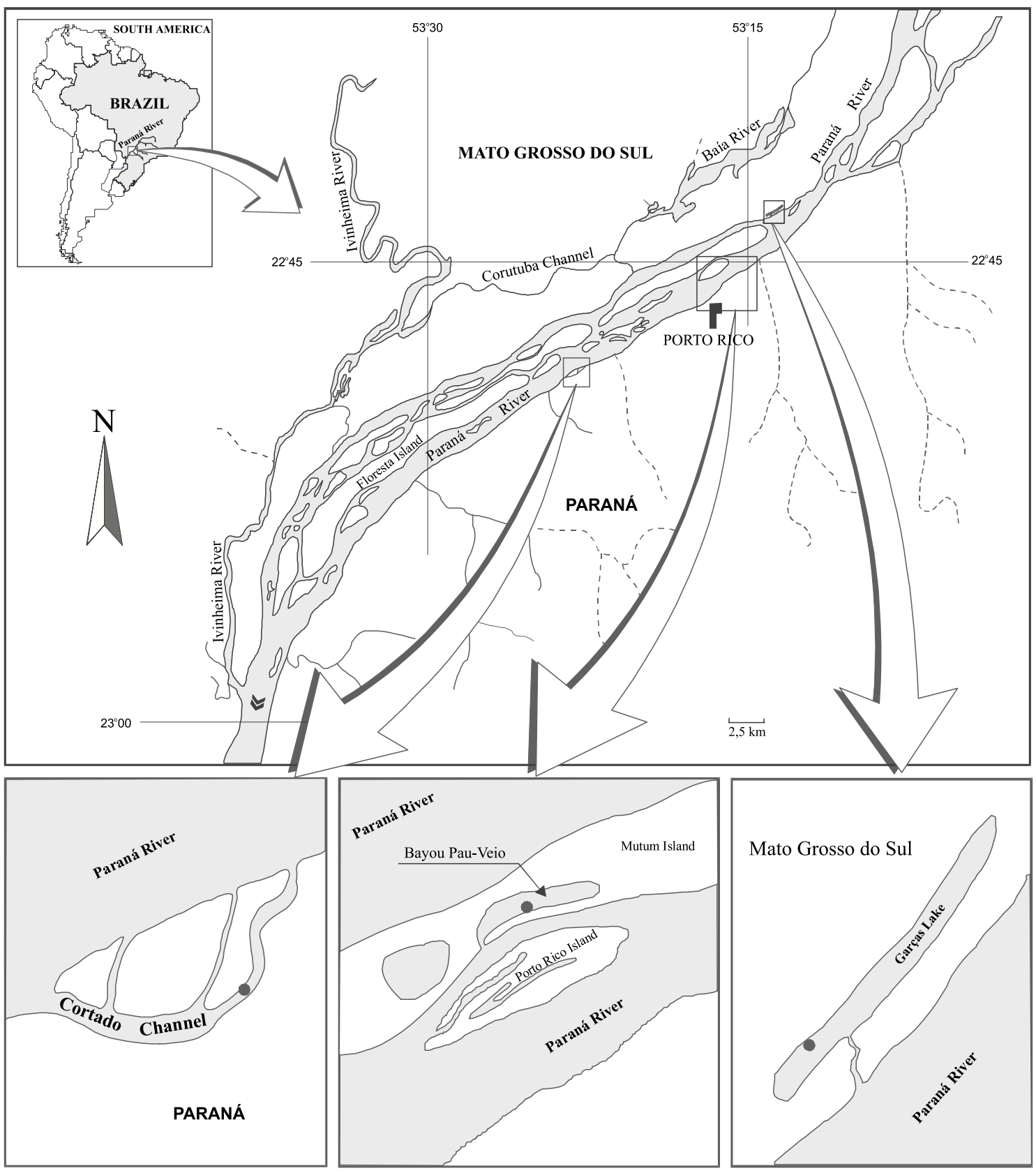

Figure 1. Locations of the three environments sampled on the Upper Paraná River floodplain (lentic system - Garças Lake; semilotic system - Pau Véio Backwater; and lotic system - Cortado Channel).

were classified according to Gleitler (1932) and Krammer \& Lange-Bertalot $(1988,1991 \mathrm{a}, \mathrm{b})$ respectively.

The similarity of the communities between systems, hydrological periods, and substratum type was measured by group analysis (UPGMA), using Dice's (Sorensen/ Czekanowski) binary index, by the program NTSYS version 1.50 (Rolf 1989).

\section{Results and Discussion}

Community richness - Taxonomic analysis of the algal periphyton community (table 2) yielded 228 taxa, 93 genera and 12 classes (30 Cyanophyceae, 34 
Table 1. Limnological parameters measured in the littoral zone of the lentic, semilotic and lotic systems during high (HW) and low water $(\mathrm{LW})$, including means and standard errors $(\mathrm{n}=3)$.

\begin{tabular}{|c|c|c|c|c|c|c|}
\hline \multirow{2}{*}{ Parameter } & \multicolumn{2}{|c|}{ Lentic System } & \multicolumn{2}{|c|}{ Semilotic System } & \multicolumn{2}{|c|}{ Lotic System } \\
\hline & HW & LW & HW & LW & HW & LW \\
\hline Water temp. $\left({ }^{\circ} \mathrm{C}\right)$ & $26.6 \pm \quad 0.6$ & $17.5 \pm 1.6$ & $26.5 \pm 0.7$ & $18.7 \pm \quad 4.3$ & $26.6 \pm \quad 0.6$ & $19.5 \pm \quad 0.8$ \\
\hline $\mathrm{pH}$ & $6.3 \pm 0.2$ & $6.7 \pm 0.1$ & $6.4 \pm 0.2$ & $7.0 \pm \quad 0.1$ & $7.0 \pm 0.01$ & $7.5 \pm \quad 0.1$ \\
\hline Conductivity $\left(\mu \mathrm{S} . \mathrm{cm}^{-1}\right)$ & $48.3 \pm 0.6$ & $50.0 \pm 3.6$ & $49.7 \pm 1.5$ & $52.0 \pm \quad 1.7$ & $57.0 \pm 1.0$ & $60.0 \pm \quad 2.0$ \\
\hline DO $\left(\mu \mathrm{g} .1^{-1}\right)$ & $3.2 \pm 2.2$ & $4.8 \pm 1.2$ & $4.4 \pm 0.9$ & $8.6 \pm 1.2$ & $8.2 \pm 0.3$ & $9.6 \pm \quad 0.4$ \\
\hline $\operatorname{Si}-\operatorname{SSR}\left(\mu \mathrm{g} .1^{-1}\right)$ & $4.9 \pm 0.7$ & $3.5 \pm 0.2$ & $5.3 \pm 0.5$ & $5.0 \pm \quad 0.5$ & $5.8 \pm 0.3$ & $5.6 \pm \quad 0.5$ \\
\hline P-PO4 $\left(\mu \mathrm{g} .1^{-1}\right)$ & $10.1 \pm 4.1$ & $1.8 \pm 0.6$ & $6.5 \pm 1.3$ & $3.2 \pm \quad 0.3$ & $5.2 \pm 1.8$ & $2.1 \pm$ \\
\hline P-PDT $\left(\mu \mathrm{g} . \mathrm{I}^{-1}\right)$ & $11.1 \pm 4.9$ & $5.3 \pm 0.4$ & $8.3 \pm 0.8$ & $3.3 \pm \quad 0.3$ & $5.6 \pm 1.4$ & $2.6 \pm \quad 0.4$ \\
\hline $\mathrm{TP}\left(\mu \mathrm{g} \cdot \mathrm{l}^{-1}\right)$ & $30.1 \pm 10.2$ & $14.8 \pm 0.4$ & $31.1 \pm 0.7$ & $48.6 \pm \quad 5.8$ & $43.5 \pm 6.3$ & $65.0 \pm 11.4$ \\
\hline $\mathrm{TN}\left(\mu \mathrm{g} . \mathrm{l}^{-1}\right)$ & $362.7 \pm 24.4$ & $539.0 \pm 8.6$ & $362.7 \pm 33.3$ & $1894.0 \pm 108.3$ & $341.3 \pm 72.2$ & $1593.7 \pm 107.1$ \\
\hline $\begin{array}{l}\text { Inorganic N:P } \\
\text { (atomic ratio) }\end{array}$ & $17 \pm 2.0$ & $93 \pm 3.7$ & $36 \pm 1.8$ & $62 \pm \quad 2.4$ & $92 \pm 3.2$ & $226 \pm \quad 0.8$ \\
\hline $\begin{array}{l}\text { TN:TP } \\
\text { (atomic ratio) }\end{array}$ & $29 \pm 1.2$ & $80 \pm 1.2$ & $26 \pm 0.8$ & $87 \pm$ & $17 \pm 0.3$ & $56 \pm$ \\
\hline
\end{tabular}

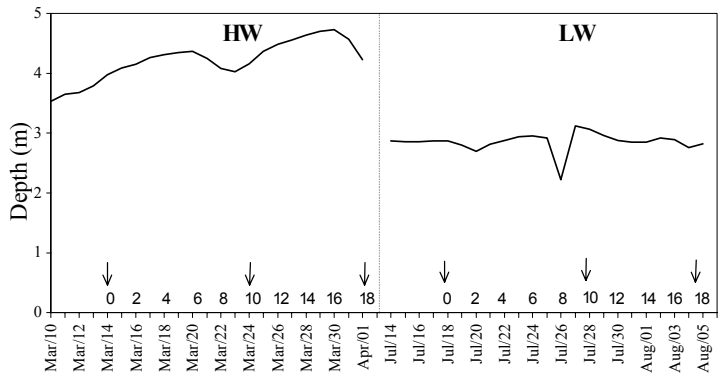

Figure 2. Daily fluctuations of the Paraná River at Porto São José city during the sampling periods (March/10 through April/1 1994, and from July/14 through August/5 1994). HW = high water; LW $=$ low water. Arrows indicate collection dates for natural substratum community; the secondary scale indicates collection dates for the artificial substratum community.

Chlorophyceae, three Ulotrichophyceae, seven Oedogoniophyceae, 62 Zygnemaphyceae, seven Euglenophyceae, one Dinophyceae, two Crysophyceae, five Xantophyceae, 74 Bacillariophyceae, one Cryptophyceae, and two Rhodophyceae).

Highest species richness was observed on artificial substratum ( $96 \%$ of the species) and during the high water stage ( $89 \%$ of the species), in contrast to results for the natural substratum (77\%) and low water stage (78\%). Of the three systems studied and comparing the same kind of substrata and hydrological periods, the lake invariably had the highest number of species, followed by the semilotic system, and last by the lotic system (table 3).

The higher species richness observed at high water, independently of environment or substratum type, was probably related to the greater availability of nutrients (table 1), mainly phosphorus, during this period (Rodrigues \& Bicudo 2001), and to greater input of propagules in the water column, which use the substrata for refuge and reproduction (Biggs 1996); and quite probably to the frequent physical disturbances of the community caused by the flood pulses. According to McCormick (1996) and Stevenson (1996), disturbances cause large changes in periphyton community structure, with disturbances of low and moderate intensity promoting the appearance of new classes, as well as species substitution.

The lake showed higher species richness, most probably for its morphometric characteristics, which lead to extensive development of aquatic macrophytes and thus the availability of a large diversity of habitats; and for the low current regime, which favors the development of both firmly or loosely attached forms. These conditions are especially favored during high water, through the substantial input of phosphorus resulting from decomposition of the flooded vegetation and resuspension of the sediment (Rodrigues \& Bicudo 2001). Although the higher orthosilicate content during high water (table 1) because of the entry of the river water into the lake, silica content did not influence richness since it was not limiting in any system studied (>0.2 mg. $\mathrm{l}^{-1}$, according to Wíllen 1991).

In regard to succession (figure 3), by the second day the community already included a high number of species (25-45 taxa), with a gradual increase in richness even in the initial stages, followed by more 
Table 2. Species list of periphytic algae by substrata, type of environment (LS = lentic system; SS = semilotic system; $\mathrm{SR}=$ lotic system), and hydrological stage ( $\mathrm{HW}=$ high water; $\mathrm{LW}=$ low water) in the Paraná River floodplain.

\begin{tabular}{|c|c|c|c|c|c|c|c|c|c|c|c|c|}
\hline \multirow[b]{3}{*}{ CYANOPHYCEAE } & \multicolumn{6}{|c|}{ Artificial substratum } & \multicolumn{6}{|c|}{ Natural substratum } \\
\hline & \multicolumn{2}{|c|}{$\begin{array}{c}\text { LS } \\
\text { HW LW }\end{array}$} & \multicolumn{2}{|c|}{$\begin{array}{c}\text { SS } \\
\text { HW LW }\end{array}$} & \multicolumn{2}{|c|}{$\begin{array}{c}\text { SR } \\
\text { HW LW }\end{array}$} & \multicolumn{2}{|c|}{$\begin{array}{c}\text { LS } \\
\text { HW LW }\end{array}$} & \multicolumn{2}{|c|}{$\begin{array}{c}\text { SS } \\
\text { HW LW }\end{array}$} & \multicolumn{2}{|c|}{$\begin{array}{c}\text { SR } \\
\text { HW LW }\end{array}$} \\
\hline & & & & & & & & & & & & \\
\hline Anabaena circinalis $\mathrm{Rab}$. & $\mathrm{x}$ & & & & $\mathrm{x}$ & $\mathrm{x}$ & $\mathrm{x}$ & & $\mathrm{x}$ & & $\mathrm{x}$ & $\mathrm{x}$ \\
\hline A. solitaria Kleb. & & & $\mathrm{x}$ & $\mathrm{x}$ & & $\mathrm{x}$ & & & & $\mathrm{x}$ & & $\mathrm{x}$ \\
\hline Anabaena sp. & $\mathrm{x}$ & $\mathrm{x}$ & & & & & $\mathrm{x}$ & & & & & \\
\hline Aphanocapsa pulchra (Kütz.) Rab. & $\mathrm{x}$ & & & $\mathrm{x}$ & & & $\mathrm{x}$ & & $\mathrm{x}$ & $\mathrm{x}$ & & \\
\hline Calothrix brevissima West & $\mathrm{x}$ & $\mathrm{x}$ & & $\mathrm{x}$ & & & $\mathrm{x}$ & $\mathrm{x}$ & & & & \\
\hline C. fusca (Kützing) Born. et Flah. & $\mathrm{x}$ & & & & & & $\mathrm{x}$ & & & & & \\
\hline Chamaesiphon sp. & & & & & & & $\mathrm{x}$ & $\mathrm{x}$ & $\mathrm{x}$ & & & \\
\hline Chroococcus minutus (Kütz.) Näg. & $\mathrm{x}$ & $\mathrm{x}$ & $\mathrm{x}$ & $\mathrm{x}$ & & & $\mathrm{x}$ & $\mathrm{x}$ & $\mathrm{x}$ & & & \\
\hline Cylindrospermum muscicola Kütz. ex Born. \& Flah. & $\mathrm{x}$ & & & & & & $\mathrm{x}$ & & $\mathrm{x}$ & & & \\
\hline Lyngbya epiphytica Hir. & $\mathrm{x}$ & & & & & & & $\mathrm{x}$ & & & & \\
\hline L. lagerheimii Gom. & $\mathrm{x}$ & & $\mathrm{x}$ & & & & $\mathrm{x}$ & & $\mathrm{x}$ & & & \\
\hline L. martensiana Men. ex Gom. & & & & & $\mathrm{x}$ & $\mathrm{x}$ & & & & & $\mathrm{x}$ & $\mathrm{x}$ \\
\hline L. nordgaardii Wille & $\mathrm{x}$ & & $\mathrm{x}$ & $\mathrm{x}$ & $\mathrm{x}$ & $\mathrm{x}$ & & & $\mathrm{x}$ & $\mathrm{x}$ & $\mathrm{x}$ & $\mathrm{x}$ \\
\hline L. pellucida Um. & & & & & $\mathrm{x}$ & & & & $\mathrm{x}$ & & & $\mathrm{x}$ \\
\hline Lyngbya sp.1 & $\mathrm{x}$ & & $\mathrm{x}$ & $\mathrm{x}$ & & & $\mathrm{x}$ & $\mathrm{x}$ & & & & \\
\hline Lyngbya sp. 2 & $\mathrm{x}$ & & & & & & $\mathrm{x}$ & $\mathrm{x}$ & & & & \\
\hline Merismopedia glauca (Ehr.) Kütz. & & & & & & $\mathrm{x}$ & & & & & & \\
\hline M. punctata Meyen & $\mathrm{x}$ & & $\mathrm{x}$ & $\mathrm{x}$ & & & $\mathrm{x}$ & & $\mathrm{x}$ & $\mathrm{x}$ & & \\
\hline Microchaete sp. & $\mathrm{x}$ & & & & $\mathrm{x}$ & & $\mathrm{x}$ & & & & $\mathrm{x}$ & $\mathrm{x}$ \\
\hline Microcoleus brasiliensis Borge & $\mathrm{x}$ & & & & & & & & & & & \\
\hline Oscillatoria amphibia Ag. ex Gom. & $\mathrm{x}$ & $\mathrm{x}$ & $\mathrm{x}$ & & & & $\mathrm{x}$ & $\mathrm{x}$ & $\mathrm{x}$ & $\mathrm{x}$ & & \\
\hline O. foreaui Frémy & & $\mathrm{x}$ & & & & & & $\mathrm{x}$ & & & & \\
\hline O. geminata Men. ex Gom. & $\mathrm{x}$ & $\mathrm{x}$ & $\mathrm{x}$ & & & & $\mathrm{x}$ & $\mathrm{x}$ & & & & \\
\hline O. limosa Ag. ex Gom. & & & $\mathrm{x}$ & & & & & & $\mathrm{x}$ & & & \\
\hline O. sancta Kütz. ex Gom. & $\mathrm{x}$ & $\mathrm{x}$ & & & & & $\mathrm{x}$ & $\mathrm{x}$ & & $\mathrm{x}$ & & \\
\hline O. splendida Grev. ex Gom. & $\mathrm{x}$ & & & & & & & & & & & \\
\hline O. subtilissima (Kütz.) Gom. & $\mathrm{x}$ & $\mathrm{x}$ & & & $\mathrm{x}$ & $\mathrm{x}$ & $\mathrm{x}$ & & & & $\mathrm{x}$ & $\mathrm{x}$ \\
\hline Pseudanabaena sp. & & & $\mathrm{x}$ & $\mathrm{x}$ & $\mathrm{x}$ & $\mathrm{x}$ & & & $\mathrm{x}$ & $\mathrm{x}$ & $\mathrm{x}$ & $\mathrm{x}$ \\
\hline Schizothrix lamyi Gom. & & & & & $\mathrm{x}$ & $\mathrm{x}$ & & & & & $\mathrm{x}$ & \\
\hline Spirulina princeps West \& West & & $\mathrm{x}$ & & & & & & & & & & \\
\hline \multicolumn{13}{|l|}{ CHLOROPHYCEAE } \\
\hline Ankistrodesmus bibraianus (Reinsch) Kors. & $\mathrm{x}$ & & & & & & $\mathrm{x}$ & & & $\mathrm{x}$ & & \\
\hline A. fusiformis Corda & $\mathrm{x}$ & & & & & & $\mathrm{x}$ & & & & & \\
\hline Aphanochaete repens A. Br. & $\mathrm{x}$ & $\mathrm{x}$ & & & $\mathrm{x}$ & & $\mathrm{x}$ & & $\mathrm{x}$ & & & \\
\hline Chaetophora elegans (Roth) Ag. & & & & & $\mathrm{x}$ & & & & & & $\mathrm{x}$ & $\mathrm{x}$ \\
\hline Characiellopsis skujae (Fott) Kom. & $\mathrm{x}$ & & $\mathrm{x}$ & $\mathrm{x}$ & & & $\mathrm{x}$ & & $\mathrm{x}$ & & & $\mathrm{x}$ \\
\hline Characium ornithocephallum A. Br. & & & $\mathrm{x}$ & & & & $\mathrm{x}$ & & $\mathrm{x}$ & & & \\
\hline Characium sp. & & & $\mathrm{x}$ & & & $\mathrm{x}$ & & & $\mathrm{x}$ & $\mathrm{x}$ & & \\
\hline Chloromonas paradoxa Korsch. & $\mathrm{x}$ & $\mathrm{x}$ & $\mathrm{x}$ & $\mathrm{x}$ & $\mathrm{x}$ & $\mathrm{x}$ & $\mathrm{x}$ & $\mathrm{x}$ & $\mathrm{x}$ & $\mathrm{x}$ & $\mathrm{x}$ & \\
\hline Chlorococcales 1 & $\mathrm{x}$ & & & & & & & & & & & \\
\hline Coleochaete $\mathrm{cf}$. orbicularis Pring. & $\mathrm{x}$ & & $\mathrm{x}$ & & $\mathrm{x}$ & & $\mathrm{x}$ & & $\mathrm{x}$ & & & \\
\hline Coelastrum cambricum Arch. & $\mathrm{x}$ & & & $\mathrm{x}$ & & & $\mathrm{x}$ & & & & & \\
\hline Crucigenia tetrapedia (Kirchn.) West \& West & & & $\mathrm{x}$ & $\mathrm{x}$ & & & & & & & & \\
\hline Crucigeniella crucifera (Wolle) Kom. & $\mathrm{x}$ & $\mathrm{x}$ & $\mathrm{x}$ & $\mathrm{x}$ & $\mathrm{x}$ & $\mathrm{x}$ & $\mathrm{x}$ & $\mathrm{x}$ & & & & \\
\hline Dictyosphaerium pulchellum Wood & $\mathrm{x}$ & $\mathrm{x}$ & & & & $\mathrm{x}$ & $\mathrm{x}$ & & & & & \\
\hline Dimorphococcus lunatus A. Br. & $\mathrm{x}$ & $\mathrm{x}$ & & & & & $\mathrm{x}$ & $\mathrm{x}$ & & & & \\
\hline Kirchneriella lunaris (Kirch.) Möb. & $\mathrm{x}$ & & & $\mathrm{x}$ & & $\mathrm{x}$ & & & & & & \\
\hline K. obesa (W. West) Schm. & $\mathrm{x}$ & $\mathrm{x}$ & $\mathrm{x}$ & & & & & & & & & \\
\hline Monoraphidium pusillum (Printz) Kom.-Legn. & $\mathrm{x}$ & & $\mathrm{x}$ & $\mathrm{x}$ & $\mathrm{x}$ & & & & $\mathrm{x}$ & $\mathrm{x}$ & $\mathrm{x}$ & \\
\hline Oocystis crassa Wittr. & $\mathrm{x}$ & $\mathrm{x}$ & $\mathrm{x}$ & $\mathrm{x}$ & $\mathrm{x}$ & $\mathrm{x}$ & $\mathrm{x}$ & $\mathrm{x}$ & & $\mathrm{x}$ & $\mathrm{x}$ & $\mathrm{x}$ \\
\hline Ophiocytium capitatum Wolle & $\mathrm{x}$ & & $\mathrm{x}$ & & & & $\mathrm{x}$ & & $\mathrm{x}$ & & & \\
\hline
\end{tabular}




\begin{tabular}{|c|c|c|c|c|c|c|c|c|c|c|c|c|}
\hline \multirow[b]{3}{*}{ O. cochleare (Eichw.) A. Br. } & \multicolumn{6}{|c|}{ Artificial substratum } & \multicolumn{6}{|c|}{ Natural substratum } \\
\hline & \multicolumn{2}{|c|}{$\begin{array}{c}\text { LS } \\
\text { HW LW }\end{array}$} & \multicolumn{2}{|c|}{$\begin{array}{c}\text { SS } \\
\text { HW LW }\end{array}$} & \multicolumn{2}{|c|}{$\begin{array}{c}\text { SR } \\
\text { HW LW }\end{array}$} & \multicolumn{2}{|c|}{$\begin{array}{c}\text { LS } \\
\text { HW LW }\end{array}$} & \multicolumn{2}{|c|}{$\begin{array}{c}\text { SS } \\
\text { HW LW }\end{array}$} & \multicolumn{2}{|c|}{$\begin{array}{c}\text { SR } \\
\text { HW LW }\end{array}$} \\
\hline & $\mathrm{x}$ & $\mathrm{x}$ & $\mathrm{x}$ & & & & $\mathrm{x}$ & & & & & \\
\hline Pediastrum duplex Meyen & & & & & & & & $\mathrm{x}$ & $\mathrm{x}$ & & & \\
\hline P. tetras (Eh.) Ralfs & $\mathrm{x}$ & $\mathrm{x}$ & $\mathrm{x}$ & $\mathrm{x}$ & & $\mathrm{x}$ & $\mathrm{x}$ & $\mathrm{x}$ & $\mathrm{x}$ & & & $\mathrm{x}$ \\
\hline Phytelios viridis Frenz. & $\mathrm{x}$ & & $\mathrm{x}$ & & & & & & & & $\mathrm{x}$ & \\
\hline Scenedesmus acuminatus (Lag.) Chod. & $\mathrm{x}$ & & $\mathrm{x}$ & $\mathrm{x}$ & & $\mathrm{x}$ & & & $\mathrm{x}$ & $\mathrm{x}$ & & \\
\hline S. bicaudatus (Hansg.) Chod. & & & & $\mathrm{x}$ & & $\mathrm{x}$ & & & & $\mathrm{x}$ & & \\
\hline S. denticulatus Lag. & $\mathrm{x}$ & $\mathrm{x}$ & & & & & $\mathrm{x}$ & $\mathrm{x}$ & $\mathrm{x}$ & $\mathrm{x}$ & & $\mathrm{x}$ \\
\hline S. ovalternus Chod. & $\mathrm{x}$ & $\mathrm{x}$ & & $\mathrm{x}$ & & $\mathrm{x}$ & & & & & & \\
\hline S. quadricauda (Turp.) Breb. sensu Chod. & $\mathrm{x}$ & $\mathrm{x}$ & $\mathrm{x}$ & $\mathrm{x}$ & $\mathrm{x}$ & $\mathrm{x}$ & $\mathrm{x}$ & $\mathrm{x}$ & $\mathrm{x}$ & $\mathrm{x}$ & & \\
\hline Schroederia sp. & $\mathrm{x}$ & & & & & & $\mathrm{x}$ & & & & & \\
\hline Sorastrum americanum (Bohl.) Schm. & $\mathrm{x}$ & $\mathrm{x}$ & & & & & & & & & & \\
\hline Stigeoclonium farctum Berth. & & & $\mathrm{x}$ & & & & & & & & & \\
\hline S. tenue (C.Ag.) Kütz. & & & & & $\mathrm{x}$ & & & & & & & \\
\hline Stigeoclonium sp. & & & & & $\mathrm{x}$ & & & & & & $\mathrm{x}$ & \\
\hline \multicolumn{13}{|l|}{ ULOTRICHOPHYCEAE } \\
\hline Ulotrix aequalis Kütz. & $\mathrm{x}$ & $\mathrm{x}$ & $\mathrm{x}$ & $\mathrm{x}$ & & & & & & & & \\
\hline U. subtilissima Rab. & & & & & $\mathrm{x}$ & $\mathrm{x}$ & & & & & & \\
\hline Uronema intermedium Bourr. & $\mathrm{x}$ & $\mathrm{x}$ & $\mathrm{x}$ & & & & $\mathrm{x}$ & & $\mathrm{x}$ & & $\mathrm{x}$ & $\mathrm{x}$ \\
\hline \multicolumn{13}{|l|}{ OEDOGONIOPHYCEAE } \\
\hline Bulbochaete sp.1 & $\mathrm{x}$ & $\mathrm{x}$ & $\mathrm{x}$ & & & & $\mathrm{x}$ & $\mathrm{x}$ & $\mathrm{x}$ & $\mathrm{x}$ & & \\
\hline Bulbochaete sp.2 & & $\mathrm{x}$ & $\mathrm{x}$ & $\mathrm{x}$ & & & $\mathrm{x}$ & $\mathrm{x}$ & $\mathrm{x}$ & $\mathrm{x}$ & & \\
\hline Oedogonium reinschii Roy & $\mathrm{x}$ & & & & & & $\mathrm{x}$ & $\mathrm{x}$ & $\mathrm{x}$ & & & \\
\hline O. warminginianum Wittr. & $\mathrm{x}$ & $\mathrm{x}$ & $\mathrm{x}$ & $\mathrm{x}$ & $\mathrm{x}$ & $\mathrm{x}$ & $\mathrm{x}$ & $\mathrm{x}$ & $\mathrm{x}$ & $\mathrm{x}$ & $\mathrm{x}$ & $\mathrm{x}$ \\
\hline Oedogonium sp.1 & $\mathrm{x}$ & $\mathrm{x}$ & $\mathrm{x}$ & $\mathrm{x}$ & & $\mathrm{x}$ & $\mathrm{x}$ & $\mathrm{x}$ & $\mathrm{x}$ & $\mathrm{x}$ & $\mathrm{x}$ & $\mathrm{x}$ \\
\hline Oedogonium sp.2 & & & $\mathrm{x}$ & & & & $\mathrm{x}$ & $\mathrm{x}$ & $\mathrm{x}$ & $\mathrm{x}$ & & \\
\hline Oedogonium sp.3 & $\mathrm{x}$ & $\mathrm{x}$ & $\mathrm{x}$ & $\mathrm{x}$ & & & $\mathrm{x}$ & $\mathrm{x}$ & $\mathrm{x}$ & $\mathrm{x}$ & $\mathrm{x}$ & \\
\hline \multicolumn{13}{|l|}{ ZYGNEMAPHYCEAE } \\
\hline Actinotaenium curcubitinum (Biss.) Teil. & $\mathrm{x}$ & & $\mathrm{x}$ & $\mathrm{x}$ & $\mathrm{x}$ & $\mathrm{x}$ & & $\mathrm{x}$ & $\mathrm{x}$ & $\mathrm{x}$ & $\mathrm{x}$ & \\
\hline A. globosum (Bulnh.) Först. ex Comp. & $\mathrm{x}$ & & & & & & & & & & & \\
\hline Arthrodesтия тисronulatus Nordst. & $\mathrm{x}$ & & $\mathrm{x}$ & & & & $\mathrm{x}$ & & $\mathrm{x}$ & & & \\
\hline Closterium jenerii Ralfs & $\mathrm{x}$ & $\mathrm{x}$ & $\mathrm{x}$ & $\mathrm{x}$ & $\mathrm{x}$ & $\mathrm{x}$ & $\mathrm{x}$ & $\mathrm{x}$ & & & & \\
\hline C. kuetzingii Bréb. & & & $\mathrm{x}$ & & $\mathrm{x}$ & & & & & & & \\
\hline C. leibleinii Kütz. ex Ralfs & & & & & $\mathrm{x}$ & $\mathrm{x}$ & & & & & & \\
\hline C. navicula Bréb. & & $\mathrm{x}$ & & $\mathrm{x}$ & & & & $\mathrm{x}$ & $\mathrm{x}$ & & $\mathrm{x}$ & \\
\hline C. parvulum Näg. & $\mathrm{x}$ & & & & & & & & & & & \\
\hline C. subulatum (Kütz.) Bréb. & & & $\mathrm{x}$ & $\mathrm{x}$ & & & & & & & & \\
\hline C. venus Kütz. ex Ralfs & $\mathrm{x}$ & & $\mathrm{x}$ & & & $\mathrm{x}$ & & & $\mathrm{x}$ & $\mathrm{x}$ & & \\
\hline Cosmarium abbreviatum Rac. & $\mathrm{x}$ & $\mathrm{x}$ & $\mathrm{x}$ & $\mathrm{x}$ & & $\mathrm{x}$ & $\mathrm{x}$ & $\mathrm{x}$ & $\mathrm{x}$ & $\mathrm{x}$ & & \\
\hline C. biretum Bréb. & & & & & & & & & $\mathrm{x}$ & & & \\
\hline C. denticulatum Borge & & & & & & & $\mathrm{x}$ & & & & & \\
\hline C. granatum Bréb. ex Ralfs & & & $\mathrm{x}$ & & & & & & $\mathrm{x}$ & & & \\
\hline C. impressulum Elfv. & $\mathrm{x}$ & $\mathrm{x}$ & & & & & $\mathrm{x}$ & $\mathrm{x}$ & & & & \\
\hline C. majae Strom & $\mathrm{x}$ & $\mathrm{x}$ & & $\mathrm{x}$ & & $\mathrm{x}$ & & & & & & \\
\hline C. margaritatum (Lund) Roy \& Biss. & $\mathrm{x}$ & $\mathrm{x}$ & $\mathrm{x}$ & $\mathrm{x}$ & & $\mathrm{x}$ & $\mathrm{x}$ & $\mathrm{x}$ & $\mathrm{x}$ & & & $\mathrm{x}$ \\
\hline C. naegelianum Bréb. & $\mathrm{x}$ & & $\mathrm{x}$ & $\mathrm{x}$ & $\mathrm{x}$ & & $\mathrm{x}$ & & & $\mathrm{x}$ & & \\
\hline C. pseudopyramidatum Lund. & $\mathrm{x}$ & $\mathrm{x}$ & $\mathrm{x}$ & $\mathrm{x}$ & $\mathrm{x}$ & & $\mathrm{x}$ & & $\mathrm{x}$ & $\mathrm{x}$ & $\mathrm{x}$ & \\
\hline C. quadrum Lund. & & $\mathrm{x}$ & & $\mathrm{x}$ & & $\mathrm{x}$ & & $\mathrm{x}$ & $\mathrm{x}$ & & & \\
\hline C. triangulare Borge & $\mathrm{x}$ & & $\mathrm{x}$ & & & & & & & & & \\
\hline Cylindrocystis brebissonii (Men.) De Bary & $\mathrm{x}$ & $\mathrm{x}$ & & & & & $\mathrm{x}$ & $\mathrm{x}$ & & & & \\
\hline Desmidium aptogonum Bréb. ex Kütz. & $\mathrm{x}$ & & & & & & $\mathrm{x}$ & & & & & \\
\hline D. baileyi (Ralfs) De Bary & $\mathrm{x}$ & $\mathrm{x}$ & $\mathrm{x}$ & & & & $\mathrm{x}$ & & & & & \\
\hline D. swartzii (Ag.) Ag. ex Ralfs & $\mathrm{x}$ & & & & & & $\mathrm{x}$ & & & & & \\
\hline Euastrum elegans (Breb.) ex Ralfs & $\mathrm{x}$ & $\mathrm{x}$ & $\mathrm{x}$ & & & & $\mathrm{x}$ & $\mathrm{x}$ & $\mathrm{x}$ & $\mathrm{x}$ & & \\
\hline E. gemmatum (Breb.) ex Ralfs & & $\mathrm{x}$ & $\mathrm{x}$ & $\mathrm{x}$ & & & & & & & & \\
\hline
\end{tabular}




\begin{tabular}{|c|c|c|c|c|c|c|c|c|c|c|c|c|}
\hline & \multicolumn{6}{|c|}{ Artificial substratum } & \multicolumn{6}{|c|}{ Natural substratum } \\
\hline & \multicolumn{2}{|c|}{$\begin{array}{c}\text { LS } \\
\text { HW LW }\end{array}$} & \multicolumn{2}{|c|}{$\begin{array}{c}\text { SS } \\
\text { HW LW }\end{array}$} & \multicolumn{2}{|c|}{$\begin{array}{c}\text { SR } \\
\text { HW LW }\end{array}$} & \multicolumn{2}{|c|}{$\begin{array}{c}\text { LS } \\
\text { HW LW }\end{array}$} & \multicolumn{2}{|c|}{$\begin{array}{c}\text { SS } \\
\text { HW LW }\end{array}$} & \multicolumn{2}{|c|}{$\begin{array}{c}\text { SR } \\
\text { HW LW }\end{array}$} \\
\hline E. mononcylum (Nord.) Rac. & $\mathrm{x}$ & & & $\mathrm{x}$ & & & & & & & & \\
\hline E.porrectum Borge & $\mathrm{x}$ & & & & & & $\mathrm{x}$ & & & & & \\
\hline E. pulchellum Bréb. & $\mathrm{x}$ & $\mathrm{x}$ & $\mathrm{x}$ & $\mathrm{x}$ & & & $\mathrm{x}$ & $\mathrm{x}$ & $\mathrm{x}$ & $\mathrm{x}$ & & \\
\hline Gonatozygon pilosum Wolle & $\mathrm{x}$ & $\mathrm{x}$ & $\mathrm{x}$ & $\mathrm{x}$ & $\mathrm{x}$ & & $\mathrm{x}$ & $\mathrm{x}$ & & & $\mathrm{x}$ & \\
\hline Hyalotheca dissiliens $(\mathrm{Sm}$.) Breb. ex Ralfs & $\mathrm{x}$ & & & & & & & & & & & \\
\hline Micrasterias arcuata Bail. & $\mathrm{x}$ & & $\mathrm{x}$ & & $\mathrm{x}$ & & $\mathrm{x}$ & & $\mathrm{x}$ & & & \\
\hline M. furcata Ag. ex Ralfs & & $\mathrm{x}$ & $\mathrm{x}$ & & $\mathrm{x}$ & & & & & & & \\
\hline M. laticeps Nordst. & $\mathrm{x}$ & & & & & & & & & & & \\
\hline M. truncata (Corda) Breb. ex Ralfs & $\mathrm{x}$ & & & & & & $\mathrm{x}$ & & & & & \\
\hline Mougeotia recurva (Hass.) De Toni & $\mathrm{x}$ & $\mathrm{x}$ & $\mathrm{x}$ & $\mathrm{x}$ & $\mathrm{x}$ & $\mathrm{x}$ & $\mathrm{x}$ & $\mathrm{x}$ & $\mathrm{x}$ & $\mathrm{x}$ & & $\mathrm{x}$ \\
\hline Netrium digitus (Ehr.) Itz. \& Rothe & $\mathrm{x}$ & & $\mathrm{x}$ & & & & $\mathrm{x}$ & $\mathrm{x}$ & $\mathrm{x}$ & & & \\
\hline Onychonema laeve Nordst. & & & $\mathrm{x}$ & & & & & & & & & \\
\hline Pleurotaenium ehrenbergii (Breb.) De Bary & & & & & & $\mathrm{x}$ & & & & & & \\
\hline P. nodosum (Bail.) Lund & & $\mathrm{x}$ & & & & & & & & & & \\
\hline Pleurotaenium sp. & & & & & & $\mathrm{x}$ & & & $\mathrm{x}$ & & & \\
\hline Spirogyra spp. & $\mathrm{x}$ & $\mathrm{x}$ & $\mathrm{x}$ & $\mathrm{x}$ & $\mathrm{x}$ & $\mathrm{x}$ & $\mathrm{x}$ & $\mathrm{x}$ & $\mathrm{x}$ & $\mathrm{x}$ & $\mathrm{x}$ & $\mathrm{x}$ \\
\hline Spondylosium panduriforme (Heim.) Teil. & $\mathrm{x}$ & $\mathrm{x}$ & $\mathrm{x}$ & & & & & $\mathrm{x}$ & & & & \\
\hline S. pulchellum Arch. & $\mathrm{x}$ & & $\mathrm{x}$ & & $\mathrm{x}$ & & $\mathrm{x}$ & & $\mathrm{x}$ & $\mathrm{x}$ & $\mathrm{x}$ & \\
\hline Staurastrum arctiscon (Ehr.) Lund. & $\mathrm{x}$ & & $\mathrm{x}$ & & & & & & & & & \\
\hline S. asterias Nyg. & $\mathrm{x}$ & & $\mathrm{x}$ & $\mathrm{x}$ & & & & & $\mathrm{x}$ & $\mathrm{x}$ & & \\
\hline S. leptacanthum Nordst. & & $\mathrm{x}$ & $\mathrm{x}$ & $\mathrm{x}$ & & & & & & & & \\
\hline S. leptocladum Nordst. & $\mathrm{x}$ & $\mathrm{x}$ & $\mathrm{x}$ & $\mathrm{x}$ & & $\mathrm{x}$ & $\mathrm{x}$ & & & & & \\
\hline S. margaritaceum Ehr. ex Ralfs & $\mathrm{x}$ & & $\mathrm{x}$ & & & & & & & & & \\
\hline S. muticum Breb. ex Ralfs & & $\mathrm{x}$ & $\mathrm{x}$ & $\mathrm{x}$ & & $\mathrm{x}$ & & $\mathrm{x}$ & $\mathrm{x}$ & $\mathrm{x}$ & $\mathrm{x}$ & \\
\hline S. nudibrachiatum Borge & & $\mathrm{x}$ & $\mathrm{x}$ & $\mathrm{x}$ & & $\mathrm{x}$ & & $\mathrm{x}$ & & $\mathrm{x}$ & & \\
\hline S. setigerum Cleve & $\mathrm{x}$ & & $\mathrm{x}$ & & & & $\mathrm{x}$ & & & & & \\
\hline S. tetracerum Ralfs & $\mathrm{x}$ & $\mathrm{x}$ & $\mathrm{x}$ & & & $\mathrm{x}$ & $\mathrm{x}$ & & & $\mathrm{x}$ & & \\
\hline S. trifidum Nordst. & $\mathrm{x}$ & $\mathrm{x}$ & $\mathrm{x}$ & $\mathrm{x}$ & & & & & $\mathrm{x}$ & $\mathrm{x}$ & & \\
\hline Staurastrum sp. & $\mathrm{x}$ & & & & & & & & & & & \\
\hline Staurodesmus cuspidatus (Breb.) Teil. & $\mathrm{x}$ & $\mathrm{x}$ & $\mathrm{x}$ & $\mathrm{x}$ & & $\mathrm{x}$ & $\mathrm{x}$ & & $\mathrm{x}$ & & & \\
\hline S. dejectus (Breb.) Teil. & & & & $\mathrm{x}$ & & & & & & $\mathrm{x}$ & & \\
\hline S. dickei (Ralfs) Lill. & $\mathrm{x}$ & $\mathrm{x}$ & & & & & & $\mathrm{x}$ & & & & \\
\hline Staurodesmus sp. & & $\mathrm{x}$ & & & & & & $\mathrm{x}$ & & & & \\
\hline Teilingia granulata (Roy \& Biss.) Bourr. & $\mathrm{x}$ & & $\mathrm{x}$ & $\mathrm{x}$ & & & $\mathrm{x}$ & & & $\mathrm{x}$ & & $\mathrm{x}$ \\
\hline Zygnema sp. & & $\mathrm{x}$ & & & & & & $\mathrm{x}$ & & & & \\
\hline \multicolumn{13}{|l|}{ EUGLENOPHYCEAE } \\
\hline Euglena acus Ehr. & $\mathrm{x}$ & $\mathrm{x}$ & $\mathrm{x}$ & $\mathrm{x}$ & & & & $\mathrm{x}$ & & $\mathrm{x}$ & & \\
\hline E. spirogyra Ehr. & & & & $\mathrm{x}$ & & & & & & $\mathrm{x}$ & & \\
\hline Phacus pleuronectes (O.F.Müll.) Duj. & $\mathrm{x}$ & & $\mathrm{x}$ & $\mathrm{x}$ & & & & & & & & \\
\hline Phacus sp. & & $\mathrm{x}$ & & & & & & & & & & \\
\hline Strombomonas gibberosa (Playf.) Defl. & $\mathrm{x}$ & & & & & & & & & & & \\
\hline Trachelomonas armata (Ehr.) Stein & $\mathrm{x}$ & $\mathrm{x}$ & $\mathrm{x}$ & & & & & & & & & \\
\hline T. rotunda Swir. emend Defl. & $\mathrm{x}$ & $\mathrm{x}$ & $\mathrm{x}$ & & & & $\mathrm{x}$ & & & & & \\
\hline \multicolumn{13}{|l|}{ DINOPHYCEAE } \\
\hline Peridinium sp. & & & $\mathrm{x}$ & $\mathrm{x}$ & & & & & $\mathrm{x}$ & $\mathrm{x}$ & & \\
\hline \multicolumn{13}{|l|}{ CHRYSOPHYCEAE } \\
\hline Dinobryon sertularia Ehr. & $\mathrm{x}$ & $\mathrm{x}$ & $\mathrm{x}$ & $\mathrm{x}$ & & & & & & & & \\
\hline Salpingoeca marsonii Lemm. & $\mathrm{x}$ & & & $\mathrm{x}$ & & & $\mathrm{x}$ & & & & & \\
\hline \multicolumn{13}{|l|}{ XANTHOPHYCEAE } \\
\hline Characiopsis aquinolaris Skuja & $\mathrm{x}$ & $\mathrm{x}$ & & & & & $\mathrm{x}$ & $\mathrm{x}$ & & & & \\
\hline C. longipes (Rab.) Borzi & & $\mathrm{x}$ & $\mathrm{x}$ & & & & $\mathrm{x}$ & $\mathrm{x}$ & & & & \\
\hline C. minor Pasch. & & & & & & & $\mathrm{x}$ & & $\mathrm{x}$ & & & \\
\hline
\end{tabular}




\begin{tabular}{|c|c|c|c|c|c|c|c|c|c|c|c|c|}
\hline \multirow[b]{3}{*}{ C. pyriformis (A. Br.) Borzi } & \multicolumn{6}{|c|}{ Artificial substratum } & \multicolumn{6}{|c|}{ Natural substratum } \\
\hline & \multicolumn{2}{|c|}{$\begin{array}{c}\text { LS } \\
\text { HW LW }\end{array}$} & \multicolumn{2}{|c|}{$\begin{array}{c}\text { SS } \\
\text { HW LW }\end{array}$} & \multicolumn{2}{|c|}{$\begin{array}{c}\text { SR } \\
\text { HW LW }\end{array}$} & \multicolumn{2}{|c|}{$\begin{array}{c}\text { LS } \\
\text { HW LW }\end{array}$} & \multicolumn{2}{|c|}{$\begin{array}{c}\text { SS } \\
\text { HW LW }\end{array}$} & \multicolumn{2}{|c|}{$\begin{array}{c}\text { SR } \\
\text { HW LV }\end{array}$} \\
\hline & $\mathrm{x}$ & $\mathrm{x}$ & $\mathrm{x}$ & $\mathrm{x}$ & & & $\mathrm{x}$ & & $\mathrm{x}$ & & & \\
\hline C. subulata (A. Br.) Borzi & $\mathrm{x}$ & & $\mathrm{x}$ & $\mathrm{x}$ & & & $\mathrm{x}$ & & $\mathrm{x}$ & & & $\mathrm{x}$ \\
\hline \multicolumn{13}{|l|}{ BACILLARIOPHYCEAE } \\
\hline Achnanthes exigua Grun. & & $\mathrm{x}$ & & & & & & & & & & \\
\hline A. inflata (Kütz.) Grun. & & $\mathrm{x}$ & & & $\mathrm{x}$ & & & & & & & \\
\hline A. minutissima Kütz. & $\mathrm{x}$ & $\mathrm{x}$ & $\mathrm{x}$ & $\mathrm{x}$ & $\mathrm{x}$ & $\mathrm{x}$ & $\mathrm{x}$ & $\mathrm{x}$ & $\mathrm{x}$ & $\mathrm{x}$ & $\mathrm{x}$ & $\mathrm{x}$ \\
\hline A. lanceolata (Breb.) Grun. & & & & & $\mathrm{x}$ & & & & & & & \\
\hline Amphipleura lindheimeri Grun. & & & & & $\mathrm{x}$ & $\mathrm{x}$ & & & & & $\mathrm{x}$ & $\mathrm{x}$ \\
\hline Anomoeoneis vitrea (Grun.) Ross & & & & & & & & $\mathrm{x}$ & & & & \\
\hline Aulacoseira ambigua (Grun.) Sim. & & & & & $\mathrm{x}$ & & & & & & $\mathrm{x}$ & \\
\hline A. distans (Ehr.) Sim. & & & $\mathrm{x}$ & $\mathrm{x}$ & $\mathrm{x}$ & $\mathrm{x}$ & & & & $\mathrm{x}$ & $\mathrm{x}$ & $\mathrm{x}$ \\
\hline A. granulata (Ehr.) Sim. & $\mathrm{x}$ & $\mathrm{x}$ & $\mathrm{x}$ & $\mathrm{x}$ & $\mathrm{x}$ & $\mathrm{x}$ & $\mathrm{x}$ & $\mathrm{x}$ & $\mathrm{x}$ & $\mathrm{x}$ & $\mathrm{x}$ & $\mathrm{x}$ \\
\hline A. herzogii (Lemm.) Sim. & $\mathrm{x}$ & $\mathrm{x}$ & & $\mathrm{x}$ & $\mathrm{x}$ & & $\mathrm{x}$ & & $\mathrm{x}$ & $\mathrm{x}$ & & \\
\hline Cocconeis placentula Ehr. & $\mathrm{x}$ & & & $\mathrm{x}$ & & & & & & & & $\mathrm{x}$ \\
\hline Cyclotella kuetzingiana Thwait. & & & & & & & & & & & $\mathrm{x}$ & \\
\hline C. meneghiniana Kütz. & & & & & & & $\mathrm{x}$ & & & & $\mathrm{x}$ & \\
\hline C. pseudostelligera Hust. & $\mathrm{x}$ & $\mathrm{x}$ & $\mathrm{x}$ & & $\mathrm{x}$ & $\mathrm{x}$ & $\mathrm{x}$ & $\mathrm{x}$ & & & & $\mathrm{x}$ \\
\hline C. stelligera Cleve \& Grun. & & $\mathrm{x}$ & $\mathrm{x}$ & $\mathrm{x}$ & $\mathrm{x}$ & & & & & $\mathrm{x}$ & $\mathrm{x}$ & \\
\hline Cymbella affinis Kütz. & $\mathrm{x}$ & & & & $\mathrm{x}$ & $\mathrm{x}$ & & & & & & $\mathrm{x}$ \\
\hline C. gracilis (Ehr.) Kütz. & & $\mathrm{x}$ & $\mathrm{x}$ & $\mathrm{x}$ & & & $\mathrm{x}$ & $\mathrm{x}$ & $\mathrm{x}$ & $\mathrm{x}$ & & \\
\hline C. mesiana Choln. & $\mathrm{x}$ & $\mathrm{x}$ & $\mathrm{x}$ & $\mathrm{x}$ & $\mathrm{x}$ & & $\mathrm{x}$ & & $\mathrm{x}$ & & $\mathrm{x}$ & $\mathrm{x}$ \\
\hline C. minuta Hilse ex Rab. & $\mathrm{x}$ & $\mathrm{x}$ & $\mathrm{x}$ & $\mathrm{x}$ & $\mathrm{x}$ & $\mathrm{x}$ & $\mathrm{x}$ & $\mathrm{x}$ & $\mathrm{x}$ & $\mathrm{x}$ & $\mathrm{x}$ & $\mathrm{x}$ \\
\hline C. silesiaca Bleisch ex Rab. & $\mathrm{x}$ & $\mathrm{x}$ & $\mathrm{x}$ & $\mathrm{x}$ & $\mathrm{x}$ & $\mathrm{x}$ & $\mathrm{x}$ & $\mathrm{x}$ & $\mathrm{x}$ & $\mathrm{x}$ & $\mathrm{x}$ & $\mathrm{x}$ \\
\hline C. tumida (Breb. ex Kütz.) Van Heurck & & & & & & $\mathrm{x}$ & & & & & & $\mathrm{x}$ \\
\hline Diploneis elliptica (Kütz.) Cleve & & & $\mathrm{x}$ & & & & & & & & & \\
\hline D. subovalis Cleve & & $\mathrm{x}$ & & $\mathrm{x}$ & & & & & & & & \\
\hline Eunotia bilunaris (Ehr.) Mills & $\mathrm{x}$ & $\mathrm{x}$ & $\mathrm{x}$ & $\mathrm{x}$ & $\mathrm{x}$ & & $\mathrm{x}$ & $\mathrm{x}$ & $\mathrm{x}$ & $\mathrm{x}$ & $\mathrm{x}$ & \\
\hline E. camelus Ehr. & & & & & & & & & & & $\mathrm{x}$ & \\
\hline E. flexuosa (Breb.) Kütz. & & & & & & & $\mathrm{x}$ & $\mathrm{x}$ & & & & \\
\hline E. incisa Greg. & $\mathrm{x}$ & $\mathrm{x}$ & & & & & $\mathrm{x}$ & $\mathrm{x}$ & & & & \\
\hline E. lineolata Hust. & $\mathrm{x}$ & $\mathrm{x}$ & $\mathrm{x}$ & & & & & $\mathrm{x}$ & $\mathrm{x}$ & $\mathrm{x}$ & & \\
\hline E. minor (Kütz.) Grun. & $\mathrm{x}$ & & $\mathrm{x}$ & & & & $\mathrm{x}$ & & $\mathrm{x}$ & & $\mathrm{x}$ & $\mathrm{x}$ \\
\hline E. pectinalis (Dillwyn) Rab. & $\mathrm{x}$ & $\mathrm{x}$ & & $\mathrm{x}$ & $\mathrm{x}$ & & $\mathrm{x}$ & $\mathrm{x}$ & & & $\mathrm{x}$ & $\mathrm{x}$ \\
\hline E. praerupta Ehr. & $\mathrm{x}$ & $\mathrm{x}$ & & & $\mathrm{x}$ & & $\mathrm{x}$ & $\mathrm{x}$ & $\mathrm{x}$ & & $\mathrm{x}$ & $\mathrm{x}$ \\
\hline E. sudetica $\mathrm{O}$. Müller & $\mathrm{x}$ & $\mathrm{x}$ & & & & & & $\mathrm{x}$ & & & & \\
\hline Fragilaria capucina Desm. & $\mathrm{x}$ & $\mathrm{x}$ & $\mathrm{x}$ & $\mathrm{x}$ & $\mathrm{x}$ & $\mathrm{x}$ & $\mathrm{x}$ & $\mathrm{x}$ & $\mathrm{x}$ & $\mathrm{x}$ & $\mathrm{x}$ & $\mathrm{x}$ \\
\hline F. goulardi (Breb.) Lange-Bert. & $\mathrm{x}$ & & $\mathrm{x}$ & & $\mathrm{x}$ & $\mathrm{x}$ & & & & & $\mathrm{x}$ & $\mathrm{x}$ \\
\hline F. ulna (Nitzsch) Lange-Bert. & $\mathrm{x}$ & $\mathrm{x}$ & $\mathrm{x}$ & $\mathrm{x}$ & $\mathrm{x}$ & $\mathrm{x}$ & $\mathrm{x}$ & $\mathrm{x}$ & $\mathrm{x}$ & $\mathrm{x}$ & $\mathrm{x}$ & $\mathrm{x}$ \\
\hline Frustulia rhomboides (Ehr.) De Toni & $\mathrm{x}$ & $\mathrm{x}$ & $\mathrm{x}$ & $\mathrm{x}$ & $\mathrm{x}$ & $\mathrm{x}$ & $\mathrm{x}$ & $\mathrm{x}$ & $\mathrm{x}$ & $\mathrm{x}$ & $\mathrm{x}$ & \\
\hline Gomphonema affine Kütz. & $\mathrm{x}$ & $\mathrm{x}$ & $\mathrm{x}$ & & $\mathrm{x}$ & $\mathrm{x}$ & $\mathrm{x}$ & $\mathrm{x}$ & $\mathrm{x}$ & & $\mathrm{x}$ & $\mathrm{x}$ \\
\hline G. angustum Ag. & $\mathrm{x}$ & & $\mathrm{x}$ & $\mathrm{x}$ & $\mathrm{x}$ & $\mathrm{x}$ & $\mathrm{x}$ & & $\mathrm{x}$ & & $\mathrm{x}$ & $\mathrm{x}$ \\
\hline G. augur Ehr. & $\mathrm{x}$ & $\mathrm{x}$ & $\mathrm{x}$ & $\mathrm{x}$ & $\mathrm{x}$ & $\mathrm{x}$ & $\mathrm{x}$ & & & & $\mathrm{x}$ & $\mathrm{x}$ \\
\hline G. gracile Ehr. & $\mathrm{x}$ & $\mathrm{x}$ & $\mathrm{x}$ & $\mathrm{x}$ & $\mathrm{x}$ & $\mathrm{x}$ & $\mathrm{x}$ & $\mathrm{x}$ & $\mathrm{x}$ & $\mathrm{x}$ & $\mathrm{x}$ & $\mathrm{x}$ \\
\hline G. parvulum (Kütz.) Kütz. & $\mathrm{x}$ & $\mathrm{x}$ & $\mathrm{x}$ & $\mathrm{x}$ & $\mathrm{x}$ & $\mathrm{x}$ & $\mathrm{x}$ & $\mathrm{x}$ & $\mathrm{x}$ & $\mathrm{x}$ & $\mathrm{x}$ & $\mathrm{x}$ \\
\hline G. subtile Ehr. & $\mathrm{x}$ & $\mathrm{x}$ & $\mathrm{x}$ & $\mathrm{x}$ & $\mathrm{x}$ & $\mathrm{x}$ & $\mathrm{x}$ & $\mathrm{x}$ & $\mathrm{x}$ & $\mathrm{x}$ & $\mathrm{x}$ & $\mathrm{x}$ \\
\hline G. truncatum Ehr. & & & & & & $\mathrm{x}$ & & & & & & $\mathrm{x}$ \\
\hline Gyrosigma nodiferum (Grun.) Reim. & & & & & $\mathrm{x}$ & $\mathrm{x}$ & & & & & & \\
\hline Hantzschia amphioxys (Ehr.) Grun. & & & & & $\mathrm{x}$ & & & & & & $\mathrm{x}$ & \\
\hline Melosira varians Ag. & & & & & $\mathrm{x}$ & $\mathrm{x}$ & & & & & $\mathrm{x}$ & $\mathrm{x}$ \\
\hline Navicula capitata Ehr. & & & & & & $\mathrm{x}$ & & & & & & \\
\hline N. constans Hust. & & & & & & $\mathrm{x}$ & & & & & & \\
\hline N. contenta Grun. & & & & $\mathrm{x}$ & & & & & & & & \\
\hline N. cryptocephala Kütz. & $\mathrm{x}$ & $\mathrm{x}$ & $\mathrm{x}$ & $\mathrm{x}$ & $\mathrm{x}$ & $\mathrm{x}$ & $\mathrm{x}$ & $\mathrm{x}$ & $\mathrm{x}$ & $\mathrm{x}$ & $\mathrm{x}$ & $\mathrm{x}$ \\
\hline N. cuspidata (Kütz.) Kütz. & & & & & & & & & & & $\mathrm{x}$ & \\
\hline N. disparilis Hust. & & & & & $\mathrm{x}$ & & & & & & & \\
\hline N. mutica Kütz. & & & $\mathrm{x}$ & $\mathrm{x}$ & & & & & & & & $\mathrm{x}$ \\
\hline
\end{tabular}




\begin{tabular}{|c|c|c|c|c|c|c|c|c|c|c|c|c|}
\hline \multirow[b]{3}{*}{ N. pupula Kütz. } & \multicolumn{6}{|c|}{ Artificial substratum } & \multicolumn{6}{|c|}{ Natural substratum } \\
\hline & \multicolumn{2}{|c|}{$\begin{array}{c}\text { LS } \\
\text { HW LW }\end{array}$} & \multicolumn{2}{|c|}{$\begin{array}{c}\text { SS } \\
\text { HW LW }\end{array}$} & \multicolumn{2}{|c|}{$\begin{array}{c}\text { SR } \\
\text { HW LW }\end{array}$} & \multicolumn{2}{|c|}{$\begin{array}{c}\text { LS } \\
\text { HW LW }\end{array}$} & \multicolumn{2}{|c|}{$\begin{array}{c}\text { SS } \\
\text { HW LW }\end{array}$} & \multicolumn{2}{|c|}{$\begin{array}{c}\text { SR } \\
\text { HW LV }\end{array}$} \\
\hline & $\mathrm{x}$ & & & $\mathrm{x}$ & $\mathrm{x}$ & $\mathrm{x}$ & & $\mathrm{x}$ & & & & \\
\hline N. radiosa Kütz. & & & & & $\mathrm{x}$ & & & & & & & \\
\hline N. schroeterii Meist. & & & & & $\mathrm{x}$ & $\mathrm{x}$ & & & & & $\mathrm{x}$ & $\mathrm{x}$ \\
\hline N. viridula (Kütz.) Ehr. & & & & & $\mathrm{x}$ & $\mathrm{x}$ & & $\mathrm{x}$ & & & & $\mathrm{x}$ \\
\hline Neidium affine (Ehr.) Cleve & & & & & $\mathrm{x}$ & & & & & & & \\
\hline Nitzschia acicularis (Kütz.) Schm. & & $\mathrm{x}$ & & & & & & & & & & \\
\hline N. amphibia Grun. & $\mathrm{x}$ & $\mathrm{x}$ & $\mathrm{x}$ & $\mathrm{x}$ & $\mathrm{x}$ & $\mathrm{x}$ & $\mathrm{x}$ & $\mathrm{x}$ & $\mathrm{x}$ & & $\mathrm{x}$ & $\mathrm{x}$ \\
\hline N. clausii Hantzsch & $\mathrm{x}$ & $\mathrm{x}$ & & & & & $\mathrm{x}$ & & & & $\mathrm{x}$ & \\
\hline N. palea (Kütz.) Schm. & $\mathrm{x}$ & $\mathrm{x}$ & $\mathrm{x}$ & $\mathrm{x}$ & $\mathrm{x}$ & $\mathrm{x}$ & $\mathrm{x}$ & $\mathrm{x}$ & $\mathrm{x}$ & $\mathrm{x}$ & $\mathrm{x}$ & $\mathrm{x}$ \\
\hline N. sigma (Kütz.) Schm. & & & $\mathrm{x}$ & $\mathrm{x}$ & $\mathrm{x}$ & $\mathrm{x}$ & & & $\mathrm{x}$ & & $\mathrm{x}$ & \\
\hline Pinnularia acrosphaeria Rab. & $\mathrm{x}$ & $\mathrm{x}$ & $\mathrm{x}$ & & & & $\mathrm{x}$ & & $\mathrm{x}$ & & & \\
\hline P. braunii (Grun.) Cleve & & $\mathrm{x}$ & $\mathrm{x}$ & $\mathrm{x}$ & & & $\mathrm{x}$ & $\mathrm{x}$ & & $\mathrm{x}$ & & \\
\hline P. divergens $\mathrm{W}$. Schm. & & & & $\mathrm{x}$ & & $\mathrm{x}$ & & & & & $\mathrm{x}$ & \\
\hline P. gibba Ehr. & & $\mathrm{x}$ & & $\mathrm{x}$ & & $\mathrm{x}$ & $\mathrm{x}$ & & & & & \\
\hline P. microstauron (Ehr.) Cleve & & $\mathrm{x}$ & $\mathrm{x}$ & $\mathrm{x}$ & & & & & & $\mathrm{x}$ & & \\
\hline P. termentina (Ehr.) Patr. & & & & $\mathrm{x}$ & & & & & & & & \\
\hline Stauroneis phoenicenteron (Nitzsch) Eh. & & & & $\mathrm{x}$ & & & & & & $\mathrm{x}$ & & \\
\hline Surirella angusta Kütz. & & $\mathrm{x}$ & & & $\mathrm{x}$ & $\mathrm{x}$ & $\mathrm{x}$ & & & & & $\mathrm{x}$ \\
\hline S. delicatissima Lew. & & $\mathrm{x}$ & & & & & & & & & & \\
\hline S. linearis Schm. & & & & & $\mathrm{x}$ & $\mathrm{x}$ & & & & & $\mathrm{x}$ & $\mathrm{x}$ \\
\hline S. tenera Greg. & $\mathrm{x}$ & $\mathrm{x}$ & & & & & & & & & & \\
\hline \multicolumn{13}{|l|}{ CRYPTOPHYCEAE } \\
\hline Protocryptomonas sp. & & $\mathrm{x}$ & & & & & & $\mathrm{x}$ & & & & \\
\hline \multicolumn{13}{|l|}{ RHODOPHYCEAE } \\
\hline Audouinella pygmaea (Kütz.) Web.-Van Bosse & & & & & $\mathrm{x}$ & & & & & & & $\mathrm{x}$ \\
\hline Compsopogon leptoclados Mont. & & & & & $\mathrm{x}$ & & & & & & & $\mathrm{x}$ \\
\hline
\end{tabular}

or less accentuated fluctuations in both high and low water periods in the lentic and semilotic environments. In the river, fluctuations were slight, except at the $8^{\text {th }}$ day of succession at low water. On the natural substrata, the variation in species number between collection days was also greater in the lentic and semilotic environments, mainly at high water (figure 3). Also the number of species on the artificial substratum closely approximated that of the epiphytic community, mainly in the lotic system.

The larger oscillations, mainly decreases in community richness with succession, occurred simultaneously in lentic and semilotic environments during high water $\left(10^{\text {th }}\right.$ and $16^{\text {th }}$ days, figure 3$)$ and out of phase during low water $\left(8^{\text {th }}\right.$ day in the river, $10^{\text {th }}$ in the semilotic, and $14^{\text {th }}$ in the lake, figure 3 ). These fluctuations were largely associated with the more pronounced oscillations of the level of the Paraná river (figure 2). At high water, the flood pulse caused a large oscillation in water level between the $6^{\text {th }}$ and $10^{\text {th }}$ days of succession, affecting mainly the periphyton communities of the more protected systems, in which the loosely attached forms were better represented, such as species of the genera Cosmarium and Scenedesmus (table 2). At the $16^{\text {th }}$ day, it is probable that the rise in water level influenced the decrease in community species richness, since its effect was observed in both lentic and semilotic systems. Very probably the rise in water level at day 10 did not cause the same response of the periphyton in terms of richness, since younger communities are supposedly more resilient (Peterson 1996).

At low water, the level of the Paraná River rose/ fell nearly a meter between the $6^{\text {th }}$ and $10^{\text {th }}$ days (figure 2 ), because of the operation of the reservoirs upstream. This event affected the riverine community immediately ( $8^{\text {th }}$ day), the semilotic community two days later $\left(10^{\text {th }}\right.$ day), and the lentic system after four days ( $14^{\text {th }}$ day) (figure 3$)$. The decrease in species richness was mainly affected by the decrease in desmids and few species of diatoms (Navicula and Pinnularia). Also, the communities on natural 
Table 3. Species richness of the periphytic algae community by type of systems, substrata $(A S=$ artificial substratum; NS $=$ natural substratum), and hydrological periods $(\mathrm{HW}=$ high water; $\mathrm{LW}=$ low water).

\begin{tabular}{lllllll}
\hline & \multicolumn{2}{c}{ Lentic System } & \multicolumn{2}{c}{ Semilotic System } & \multicolumn{2}{c}{ Lotic System } \\
\cline { 2 - 7 } & AS & NS & AS & NS & AS & NS \\
\hline HW & 137 & 109 & 112 & 83 & 76 & 61 \\
LW & 105 & 76 & 93 & 63 & 74 & 56 \\
\hline
\end{tabular}

Garças Lake

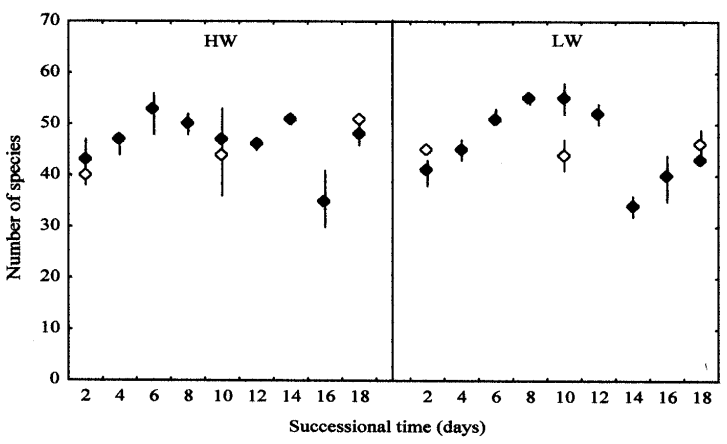

Pau Véio Backwater

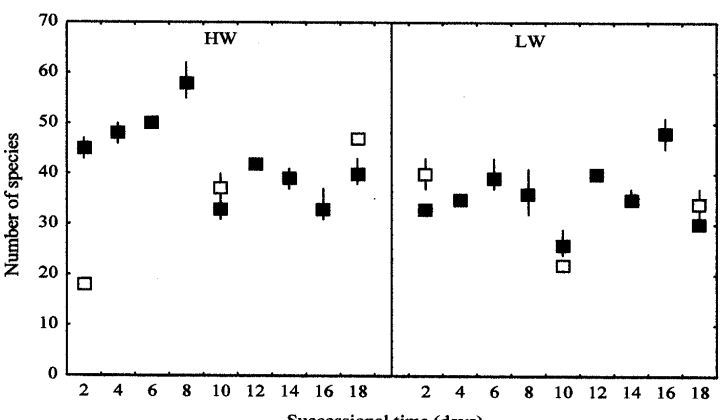

Cortado Channel

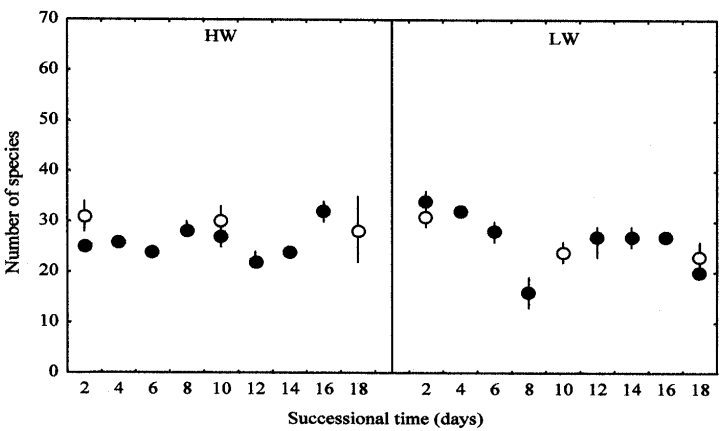

Figure 3. Mean and standard deviation $(n=2)$ in community species number on artificial (full) and natural substrata (empty) during high water (HW) and low water (LW) in lentic, semilotic, and lotic systems.

substrata, particularly those of the semilotic and lotic systems, showed a loss in species richness (figure 3, second collection). The time difference in the communities responses in different systems could be demonstrated only on the artificial substratum, by means of collections made at short intervals. Such differences in phase must have occurred on natural substratum as well, since during low water the systems show more individualized characteristics, associated with the lower degree of connection to the Paraná River (Rodrigues \& Bicudo 2001).

In general, periphyton community richness on the two substratum types in the lotic system was more stable over both macro- and micro- time scales. This lower amplitude of oscillation in species number must result from the composition of the riverine community, which includes more firmly attached forms, which are highly resistant to disturbances (Peterson 1996), such as Oedogonium reinschii Roy, O. warminginianum Wittr. and Oedogonium spp., as well as from the greater temporal homogeneity of the abiotic characteristics of the system (Rodrigues \& Bicudo 2001).

Taxonomical composition - Analysis of the taxa numbers per class and by type of environment, substratum, and hydrological period demonstrated the predominance of Zygnemaphyceae and Bacillariophyceae (figure 4, table 4). Lower species richness of Zygnemaphyceae occurred in the river, and higher richness of diatoms in the lotic and semilotic environments.

Diatoms, present in all systems and at both periods (table 2, figure 4), are rapid and efficient colonizers, capable of occupying substrata within a single day to several weeks (Hoagland et al. 1986). Moreover, many diatoms have specialized structures for fixation to substratum, such as short or long mucilaginous peduncles as in species of Gomphonema, production of mucilaginous matrices as in Cymbella, Frustulia, and Navicula, and staror branch-shaped colonies fixed at the base as in Eunotia and Fragilaria (Round 1991). The higher richness of this group in lotic system (figure 4) is partly related to these specialized structures that confer a competitive advantage on diatoms in more 
stressful ambient conditions imposed by current speed and discharge (Biggs 1996). Additionally, their small size and ability to secrete mucilage permits diatoms to fix rapidly onto smaller surfaces which are inaccessible to larger competitors (Biggs \& Hickey 1994). Although the river had higher reactive silicate content than the other two systems studied (table 1), silicate was always above the diatom growth limit $\left(>0.2 \mathrm{mg} . \mathrm{l}^{-1}\right)$.

Desmids were well represented in species mainly in the lake, followed by semilotic system (figure 4, table 2,4). True planktonic desmids are rare and most of them make use of a substratum at least for reproduction (Coesel 1996). Accordingly, higher desmid richness was related to the presence of stands of macrophytes and the positioning of the artificial substrata next to them, since the stands are important sources of algae, especially desmids (Brook 1981, Coesel 1996). The low current speeds in these systems is another probable factor in the high representation of desmids.

More even partitioning of the algal classes occurred in the lake, followed by the semilotic and lastly the lotic system (figure 4). Also, some classes were found only in certain systems and/or periods. For instance, Cryptophyceae occurred only in the lentic environment at low water, Rhodophyceae in the lotic system, and Dinophyceae in the semilotic system (tables 2, 4).

Floristic similarity in the cluster analysis among periphyton communities of the different types of system, substrata, and hydrological periods (figure 5) showed that two large groups ( $43 \%$ similarity level) were formed which discriminated the periphyton community of the lotic system (D) from the remaining environments (A-C). Within this second group, three subgroups ( $>60 \%$ similarity) were formed, corresponding to: (A) communities at high water (lake

Table 4. Algal classes species richness of the periphyton by type of environment, substrata $(\mathrm{AS}=$ artificial substratum, NS $=$ natural substratum), and hydrological periods ( $\mathrm{HW}=$ high water; $\mathrm{LW}=$ low water).

\begin{tabular}{|c|c|c|c|c|c|c|c|}
\hline & & \multicolumn{2}{|c|}{ Lentic System } & \multicolumn{2}{|c|}{ Semilotic System } & \multicolumn{2}{|c|}{ Lotic System } \\
\hline & & AS & NS & AS & NS & AS & $\mathrm{NS}$ \\
\hline \multirow{2}{*}{ Cyanophyceae } & HW & 20 & 17 & 10 & 12 & 8 & 7 \\
\hline & LW & 9 & 10 & 8 & 7 & 8 & 8 \\
\hline \multirow{2}{*}{ Chlorophyceae } & HW & 23 & 16 & 15 & 12 & 10 & 6 \\
\hline & LW & 12 & 8 & 13 & 9 & 11 & 5 \\
\hline \multirow{2}{*}{ Ulotrichophyceae } & HW & 2 & 1 & 2 & 1 & 1 & 1 \\
\hline & LW & 2 & 0 & 1 & 0 & 1 & 1 \\
\hline \multirow{2}{*}{ Oedogoniophyceae } & HW & 5 & 7 & 6 & 7 & 1 & 3 \\
\hline & LW & 5 & 7 & 4 & 6 & 2 & 2 \\
\hline \multirow{2}{*}{ Zygnemaphyceae } & HW & 42 & 27 & 37 & 22 & 12 & 7 \\
\hline & LW & 29 & 20 & 25 & 17 & 17 & 4 \\
\hline \multirow{2}{*}{ Euglenophyceae } & HW & 5 & 1 & 4 & 0 & 0 & 0 \\
\hline & LW & 4 & 0 & 0 & 2 & 0 & 0 \\
\hline \multirow{2}{*}{ Dinophyceae } & HW & 0 & 0 & 1 & 0 & 1 & 0 \\
\hline & LW & 0 & 0 & 1 & 0 & 1 & 0 \\
\hline \multirow{2}{*}{ Chrysophyceae } & HW & 2 & 1 & 1 & 0 & 0 & 0 \\
\hline & LW & 1 & 0 & 2 & 0 & 0 & 0 \\
\hline \multirow{2}{*}{ Xanthophyceae } & HW & 5 & 7 & 5 & 4 & 0 & 0 \\
\hline & LW & 4 & 2 & 2 & 0 & 0 & 1 \\
\hline \multirow{2}{*}{ Bacillariophyceae } & HW & 33 & 32 & 31 & 24 & 42 & 37 \\
\hline & LW & 38 & 27 & 34 & 21 & 35 & 33 \\
\hline \multirow{2}{*}{ Cryptophyceae } & HW & 0 & 0 & 0 & 0 & 0 & 0 \\
\hline & LW & 1 & 1 & 0 & 0 & 0 & 0 \\
\hline \multirow{2}{*}{ Rhodophyceae } & HW & 0 & 0 & 2 & 0 & 0 & 0 \\
\hline & LW & 0 & 0 & 0 & 0 & 0 & 2 \\
\hline
\end{tabular}



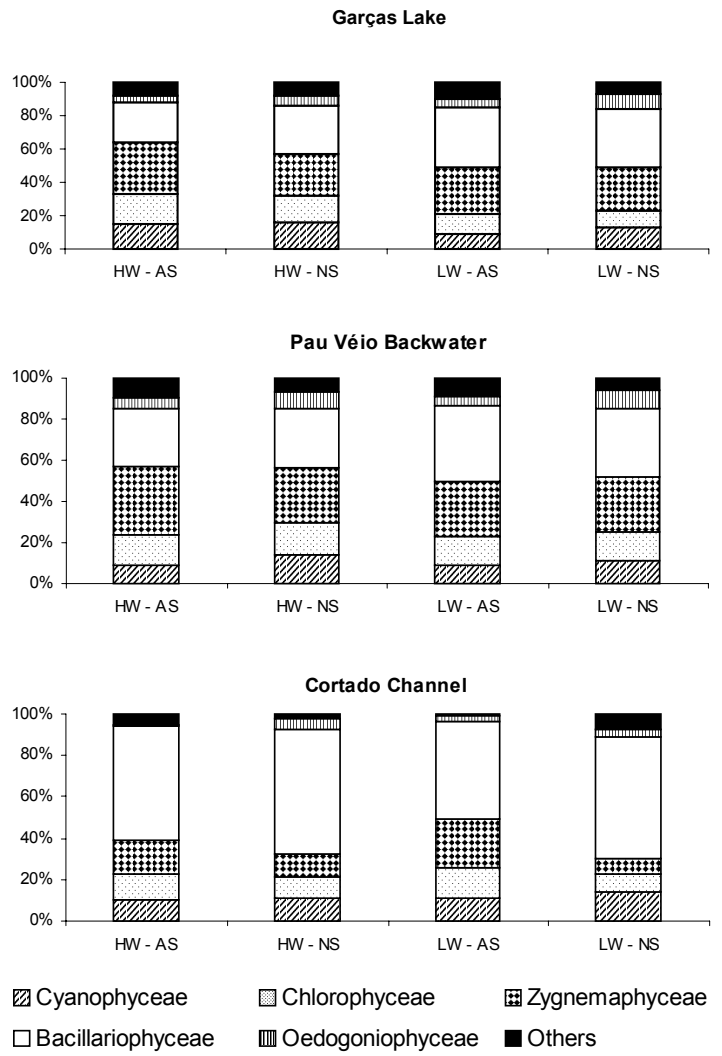

Figure 4. Relative contribution of the main algal classes to total species richness in each environment, substratum (AS: artificial substratum; NS: natural substratum), and hydrological period (HW: high water, LW: low water stages).

Similarity (Dice's index)

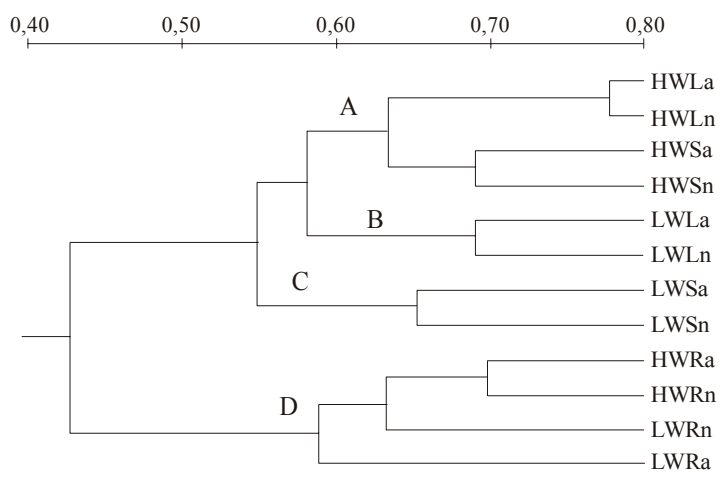

Figure 5. Dendrogram of similarity (Dice's index) between periphyton communities in different systems, substrata, and hydrological periods. Abbreviations: $\mathrm{HW}=$ high water; $\mathrm{LW}=$ low water; $\mathrm{L}=$ lentic environment; $\mathrm{S}=$ semilotic environment; $\mathrm{R}=$ lotic environment; $\mathrm{a}=$ artificial substratum; $\mathrm{n}=$ natural substratum. and semilotic environments), (B) communities at low water in the lake, and $(\mathrm{C})$ communities at low water in semilotic system. In fact, periphyton river community was less rich than in the other systems (table 2), more stable in species number during succession (figure 2,3 ), and remarkably richer on diatoms (table 4). It can also be seen that during high water, communities from both lentic and semilotic systems become more similar (> 60\% similarity) than during low water (ca. $55 \%)$. These results are in accordance with Rodrigues $\&$ Bicudo (2001) that, based on limnological abiotic characteristics of the same systems, found that lentic and semilotic systems became more similar during high water phase, and presented more individualized characteristics during low water associated with the lower degree of connection to the Paraná River. So the hypothesis put forth by Thomaz et al. (1997) referring to the homogenizing effect of the floods on the aquatic environments influenced by the upper Paraná River is also reinforced by floristic similarity among periphyton communities. Moreover, the authors observed a rupture in the behavior of the lagoons and semilotic environments, which become more homogeneous when the hydrological level is above $3.5 \mathrm{~m}$. At present higher similarity was observed when the hydrological levels of the Paraná River were above $3.5 \mathrm{~m}$ (figure 2).

Considering artificial and natural substrata (figure 5), floristic similarity between periphyton communities was quite high, about $65 \%$ (LW) and $70 \%$ (HW) for semilotic system, 70\% (LW) and $78 \%$ (HW) for the lake. River communities were less similar during low water (ca. $60 \%$ ), but became more similar during high water $(70 \%)$. So higher similarity levels were observed by substratum type, particularly during high water, indicating the homogenizing effect of the floods even on micro-spatial level.

In conclusion, floristic composition of periphyton community was mainly influenced by environment type, with a clear separation of the river community (43\% similarity level) and the communities of the other two systems (55\% similarity level). The hydrological period was next in importance and lastly, the substratum type. So, taxonomic composition of the epiphytic community was quite well represented by artificial substratum.

Although the hydrological regime is considered the main functioning force in the structure and function of river-floodplain systems (e.g. Junk 1996, Neiff 1996), the type of the system (hydrodynamic 
regime, morphometric characteristics, the degree of connection of the systems with the Paraná River) exerted a greater influence on the species composition and richness of periphytic community in the Paraná River floodplain. Therefore, the magnitude of the influence of the hydrological regime on community diversity is very probably regulated by the type of the system.

Furthermore the importance of physical disturbances in community richness was suggested, mainly of the flood pulses and the operation of the reservoir floodgates upstream. High species richness was observed even in the initial stages of colonization of the artificial substratum, indicating the high diversity of periphytic algae in the littoral zones of this floodplain. Finally, more effort is needed to evaluate the anthropogenic impact of artifical control of river discharge on the diversity changes of periphyton communities of river-floodplain systems.

Acknowledgements: We thank Dr. Carlos E. de M. Bicudo, Dr. Célia L. Sant'Anna, and Dr. Orlando Necchi Junior for their inestimable assistance in the identification of taxa of desmids, Cyanophyceae, and Rhodophyceae, respectively. Dr. Janet W. Reid translated the manuscript into English.

\section{References}

AGOSTINHO, A.A., \& ZALEWSKI, M. 1996. A planície alagável do Alto Rio Paraná: importância e preservação. Editora da Universidade Estadual de Maringá, Maringá.

BICUDO, D.C., NECCHI-JUNIOR, O. \& CHAMIXAES, B.C.B. 1995. Periphyton studies in Brazil: present status and perspectives. In Limnology in Brazil (J.G. Tundisi, C.E.M. Bicudo \& T. Matsumura-Tundisi, eds.). Academia Brasileira de Ciências/Sociedade Brasileira de Limnologia, Rio de Janeiro, p.37-58.

BIGGS, B.J.F. 1996. Patterns in benthic algal of streams. In Algal ecology; freshwater benthic ecosystems (R.J. Stevenson, M.L. Bothwell \& R.L. Lowe, eds.). Academic Press, San Diego, p.31-56.

BIGGS, B.J.F. \& C.W. HICKEY. 1994. Periphyton responses to a hydraulic gradient in a regulated river in New Zealand. Freshwater Biology 32:49-59.

BOURRELLY, P. 1975. Les algues d'eau douce: initiation à la sistematique. 1. Les algues vertes. Editions N. Boubée \& Cie., Paris.

BOURRELLY, P. 1981. Les algues d'eau douce: initiation à la sistematique. 2. Les algues jaunes et brunes, chrysophycées, xanthophycées et diatomées. Editions N. Boubée \& Cie., Paris.

BROOK, A.J. 1981. The biology of desmids. Blackwell Scientific Publishers, London.

CATTANEO, A. \& AMIREAULT, M.C. 1992. How artificial are artificial substrates for periphyton? Journal North American Benthological Society 11(2):244-256.
COESEL, P.F.M. 1996. Biogeography of desmids. Hydrobiologia 336:41-53.

GLEITLER, L. 1932. Cyanophyceae. In Kryptogamenflora von Deutschlands, Österreichs und der Schweiz (L. Rabenhorst, ed.). Akademische Verlagsgesellschaft, Leipzig, p.1-1196.

GOLDSBOROUGH, L.G. \& ROBINSON, G.G.C. 1996. Pattern in wetlands. In Algal ecology, freshwater benthic ecosystems (R.J. Stevenson, M.L. Bothwell \& R.L. Lowe, eds.). Academic Press, San Diego, p.78-117.

HOAGLAND, K.D., ZLOTSKY, A. \& PETERSON, C.G. 1986. The source of algal colonizer on rock substrates in a freshwater impoundment. In Algal biofouling (L.V. Evans \& K.D. Hoagland, eds.). Elsevier Science Publishing Company Inc., Amsterdam, p.21-39.

JATI, S. \& TRAIN, S. 1993. Representantes do gênero Trachelomonas Ehrenberg de duas lagoas da ilha Porto Rico, município de Porto Rico, Paraná, Brasil. Revista Unimar 15:37-51.

JUNK, W.J. 1996. Ecology of floodplains: a challenge for tropical limnology. In Perspectives in tropical limnology (F. Schiemer \& K.T. Boland, eds.). Academic Publishing, New York, p.255-265.

JUNK, W.J. \& DA SILVA, C.J. 1995. Neotropical floodplains: a comparison between the pantanal of Mato Grosso and the large Amazonian river floodplain. In Limnology in Brazil (J.G. Tundisi, C.E.M. Bicudo \& T. MatsumuraTundisi, eds.). Academia Brasileira de Ciências/Sociedade Brasileira de Limnologia, Rio de Janeiro, p.195-218.

KRAMMER L. \& LANGE-BERTALOT, H. 1988. Bacillariophyceae, 2: Bacillariaceae, Epithemiaceae, Surirellaceae. In Süsswasserflora von Mitteleuropa (H. Ettl, J. Gerloff, H. Heynig \& D. Mollrnhsuer, eds.). G. Fischer Verlag, Stuttgart, v. 2, pars 2, p.1-596.

KRAMMER，L. \& LANGE-BERTALOT， H. 1991a. Bacillariophyceae, 3: Centrales, Fragilariaceae, Eunotiaceae. In Süsswasserflora von Mitteleuropa (H. Ettl, J. Gerloff, H. Heynig \& D. Mollrnhsuer, eds.). G. Fischer Verlag, Stuttgart, v. 2, pars 3, p.1-576.

KRAMMER, L. \& LANGE-BERTALOT, H. $1991 \mathrm{~b}$. Bacillariophyceae, 4: Achnanthaceae: Kritische Erganzungen zu Navicula (Lineolatae) und Gomphonema. In Süsswasserflora von Mitteleuropa (H. Ettl, J. Gerloff, H. Heynig \& D. Mollrnhsuer, eds.). G. Fischer Verlag, Stuttgart, v. 2, pars 4, p.1-437.

McCORMICK, P.V. 1996. Resource competition and species coexistence in freshwater benthic algal assemblages. In Algal ecology; freshwater benthic ecosystems (R.J. Stevenson, M.L. Bothwell \& R.L. Lowe, eds.). Academic Press, San Diego, p.229-252.

NEIFF, J.J. 1996. Large rivers of South America: toward the new approach. Verhandlungen der Internationalen Vereinigung für Theoretische und Angewandte Limnologie 26:167-180.

PETERSON, C.G. 1996. Response of benthic algal communities to natural physical disturbance. In Algal ecology, freshwater benthic ecosystems (R.J. Stevenson, M.L. Bothwell \& R.L. Lowe, eds.). Academic Press, San Diego, p.375-402.

RODRIGUES, L. \& BICUDO, D. C. 2001. Limnological characteristics comparison in three systems with different hydrodynamic regime in the upper Paraná river floodplain. Acta Limnologica Brasiliensia 13: in press. 
ROLF, F.J. 1989. NTSYS-PC. Numerical taxonomy and multivariate analysis system, version 1.50. Exeter Publ. Ltd. New York.

ROUND, F.E. 1968. The biology of the algae. Edward Arnold Publishers Ltd., London.

ROUND, F.E. 1971. The taxonomy of the Chlorophyta II. British Phycological Journal 6(2):235-264.

ROUND, F.E. 1991. Diatoms in river water-monitoring studies. Journal of Applied Phycology 3:129-145.

SIMONSEN, R. 1974. The diatoms plankton of the Indian Ocean Expedition of R. V. Meteor, 1964-5. Meteor Forschungsergeb. Reihe D-Biologie 19:1-66.

STEVENSON, R.J. 1996. The stimulation and drag of current. In Algal ecology, freshwater benthic ecosystems (R.J. Stevenson, M.L. Bothwell \& R.L. Lowe, eds.). Academic Press, San Diego, p.321-340.

UTERMÖHL, H. 1958. Zur Vervollkommung der quantitativen Phytoplankton-Methodik. Mitteilungen Internationale Vereiningung fuer Theoretische und Angewandte Limnologie 9:1-38.
THOMAZ, S.M., ROBERTO, M.C. \& BINI, L.M. 1997. Caracterização limnológica dos ambientes aquáticos e influência dos níveis fluviométricos. In A planície de inundação do alto rio Paraná (A.E.A.M. Vazzoler, A.A. Agostinho \& N.S. Hahn, eds.). Editora da Universidade Estadual de Maringá, Maringá, p.73-102.

TRAIN, S. \& RODRIGUES, L.C. 1997. Fitoplâncton da planície de inundação do Alto Rio Paraná. In A planície de Inundação do alto rio Paraná (A.E.A.M. Vazzoler, A.A. Agostinho \& N.S. Hahn, eds.). Editora da Universidade Estadual de Maringá, Maringá, p.105115 .

VAZZOLER, A.E.A.M., AGOSTINHO, A.A. \& HAHN, N.S. 1997. A planície de inundação do alto rio Paraná. Editora da Universidade Estadual de Maringá, Maringá.

WÍLLEN, E. 1991. Planktonic diatoms, an ecological review. Algological Studies 62:69-106. 